\title{
The effects of COVID-19 on sickness of medical staff across departments: A single centre experience
}

\author{
Authors: Reza Khorasanee, ${ }^{A}$ Tomas Grundy, ${ }^{A}$ Alexander Isted ${ }^{A}$ and Richard Breeze ${ }^{B}$
}

\section{Introduction}

COVID-19 presents a risk to healthcare workers, incurring harm to staff physical and mental wellbeing and difficulties in provision of care and service planning.

\section{Methodology}

Doctors' anonymised demographic and staff sickness data were collected between 16 March and 26 April 2020, corresponding with the single centre's greatest COVID-19 caseload.

\section{Findings}

$128(39 \%)$ of doctors experienced at least one sickness episode. Episodes totalled 1,240 days, equating to a sickness absence rate of $9.1 \%$. Rates varied between departments and grades. High levels of sickness were seen in medicine and both adult and paediatric emergency departments with the lowest levels seen in intensive care.

Discussion

COVID-19 caused a burden of sickness on the medical workforce which must be accounted for in future workforce planning. The disparity in sickness rates across departments is likely to be multi-factorial. Further study is needed to investigate these factors to protect healthcare staff and their patients.

KEYWORDS: SARS-COV-2, coronavirus, COVID-19, staff sickness, staff absence

DOI: $10.7861 /$ clinmed.2020-0547

\section{Summary}

What is known?

COVID-19 has inflicted a significant burden of morbidity and mortality on healthcare workers, widely reported in the media. Direct illness and self-isolation rules have resulted in the need for management and staff to adapt working patterns to provide safe and consistent cover.

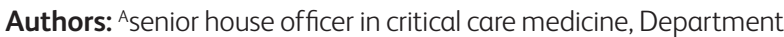
of Intensive Care, University Hospital Lewisham, London, UK; ${ }^{B}$ consultant in critical care medicine and anaesthesia, Department of Intensive Care, University Hospital Lewisham, London, UK

\section{What is the question?}

What levels of medical staff sickness were experienced during the first wave of COVID-19, were there differences between departments and grades, and how should we use this information to prepare for further waves?

\section{What was found?}

Sickness prevalence and absence rates were significantly higher than pre-pandemic and there was significant variation in sickness rates between specialties.

What is the implication for practice now? Hospitals, with the benefit of hindsight, must now prepare for further waves of COVID-19 to protect staff and patients data such as these may help in workforce planning to ensure appropriate redundancy and to also maintain key non-COVID related services.

\section{Background}

Coronavirus disease 2019 (COVID-19) has spread rapidly around the globe and was declared a pandemic by the World Health Organization (WHO) in March 2020.1 It poses unique challenges, both due to the impact it has on health systems and the degree of personal risk it places upon those who work in healthcare. China reported $3.8 \%$ of confirmed cases to be in healthcare workers and estimates have ranged from $9 \%$ to $26 \%$ in Europe. ${ }^{2}$

In the UK, many healthcare workers have contracted the virus and tragically more than 400 died in $2020,{ }^{3}$ resulting understandably in a great deal of concern among NHS staff. A British Medical Association (BMA) snapshot survey found almost one in five doctors did not feel safely protected at work during the COVID-19 pandemic. ${ }^{4}$ In an NHS already struggling with thousands of vacancies ${ }^{5}$ it is imperative to identify the factors resulting in staff sickness and to take steps to prevent morbidity and mortality among the staff responding to the COVID-19 pandemic.

England's NHS sickness absence rate, the average daily unavailability due to sickness, was $4.8 \%$ in January 2020 , with a rate of $1.5 \%$ among doctors. ${ }^{6}$ As of 2 April 2020 , the health and social care secretary, Matt Hancock, announced a $5.7 \%$ sickness rate in doctors in England due to COVID-19,7 although a one-day Royal College of Physicians poll put the rate in London at $21.5 \% .{ }^{8}$ University Hospital Lewisham is a district general hospital (DGH) in south London. We sought to quantify the impact of COVID-19 on sickness rates among doctors during the first wave to guide future workforce planning. 


\section{Methods}

This retrospective, observational study included anonymised sickness absence data provided by departmental rota coordinators in a single DGH. The departments of interest included general medicine (including care of the elderly), adult emergency department (ED), paediatric ED, anaesthetics, intensive care (ICU), general surgery, ear nose and throat surgery (ENT), and trauma and orthopaedic surgery (T\&O) for the 6-week period 16 March 2020 to 26 April 2020, corresponding to the 'first wave' of the COVID-19 pandemic in this region. ${ }^{9}$ All doctors working clinically in these departments were included. Anonymised data were collected for audit purposes and as such ethical approval was not required.

Episodes of sickness absence included self-isolation or illness due to COVID-19 symptoms, or due to symptoms in a household contact, as well as non-COVID related sickness, and are hereafter referred to simply as 'sickness episodes'. Each individual sickness episode was recorded by specialty, grade ('consultants', including associate specialists, and non-consultant grades, hereafter referred to as 'junior doctors'), and duration only. Each department reported basic pooled demographic data of all its doctors including sex, ethnicity, and grade but demographic or other identifiable data attached to individual sickness episodes were not collected to ensure staff anonymity.

Doctors unable to perform 'frontline' clinical duties due to unacceptable risk due to pre-existing health issues were excluded. If a sickness episode started or finished outside the 6-week period but part of it fell within the period then the full duration of that episode was counted.

The primary outcomes were sickness prevalence and the sickness absence rate. Sickness prevalence was defined as the proportion of doctors that had one or more sickness episode within the period of interest. Sickness absence rate was calculated by dividing the total sickness absence days including non-working days by the total days available for each member of staff over this period ( 42 days). These outcomes were compared between individual departments and between consultants and junior doctors.

Data are presented as number (percentage) or median (interquartile range) as appropriate. All data were considered nonparametric in nature and $\chi^{2}$, Mann-Whitney $U$, and Kruskal-Wallis tests were used as appropriate. $\mathrm{P}<0.05$ was deemed statistically significant. All analysis was performed using IBM SPSS for Macintosh version 26.

\section{Results}

Across the eight departments of interest, 326 doctors worked clinically during a 6 -week period. 84 (25.8\%) were consultants and $242(74.2 \%)$ were junior doctors. 165 (50.6\%) were male and 161 (49.4\%) were female. 166 (50.9\%) had a black, Asian or minority ethnic (BAME) background, and 160 (49.1\%) were white. Variation in staff demographics and seniority between departments are detailed in supplementary material S1.

The sickness prevalence was 39.0\% (127/326 doctors experienced at least one sickness episode during this period), with 138 episodes in total. The median and modal duration of these episodes was 7.0 (IQR 7.0-11.8) days. These episodes totalled 1240 days which equates to a sickness absence rate of $9.1 \%$.

There was significant variance in sickness prevalence between departments $(p=0.044)$, with the highest levels seen in medicine $(49.0 \%)$ and the lowest level seen in ICU (17.1\%). There was a range of sickness prevalence across other departments; adult ED (43.5\%), paediatric ED (39.1\%), anaesthetics (38.3\%), general surgery (33.3\%), T\&O (29.4\%) and ENT (26.1\%) (Fig 1a).

Sickness absence rates varied from $12.0 \%$ in medicine to $3.7 \%$ in ICU $(p=0.004)$. Rates in other departments were adult ED $(8.6 \%)$, paediatric ED (7.1\%), anaesthetics $(9.7 \%)$, general surgery $(10.5 \%)$, T\&O (7.4\%), and ENT (5.9\%) (Fig 1b). a) Sickness prevalence $\%$ by specialty

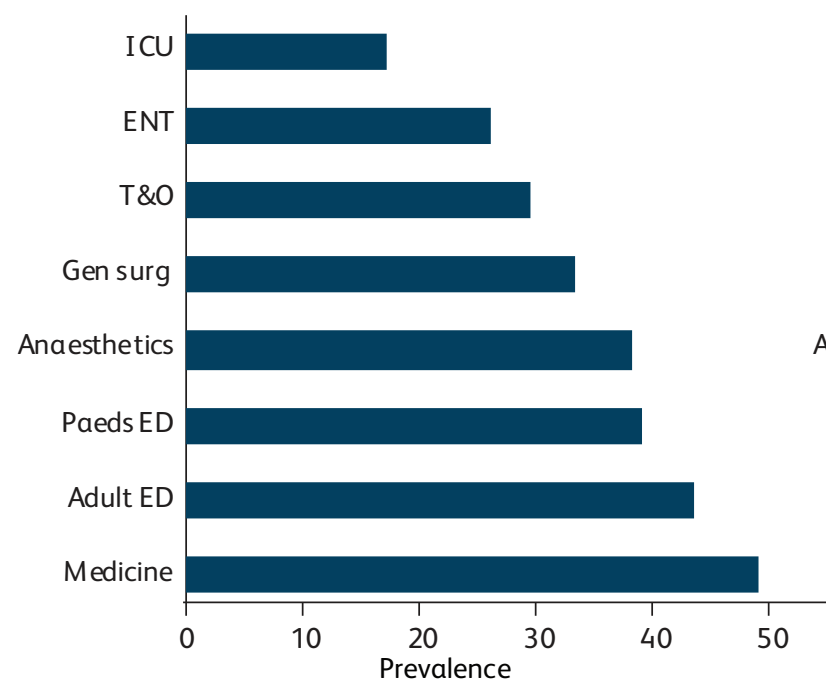

b) Sickness absence rate by specialty

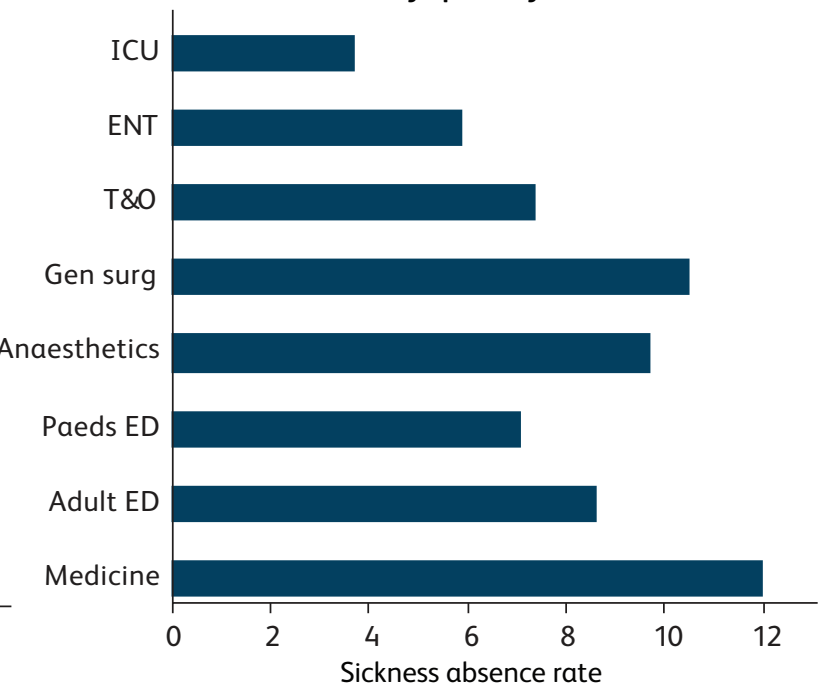

Fig 1. Sickness prevalence and sickness absence rate by speciality. (a) Sickness prevalence by specialty (defined as the proportion of doctors that had one or more sickness episode within the period of interest). (b) Sickness absence rate by specialty (defined as the total sickness absence days including non-working days divided by the total days available for each member of staff over this period ( 42 days). Adult ED = adult emergency department; ENT $=$ ear, nose and throat surgery; ICU = department of intensive care; gen surg = general surgery; medicine = department of medicine and care of the elderly: paeds $E D=$ paediatric emergency department; $T \& O=$ trauma and orthopaedic surgery. 


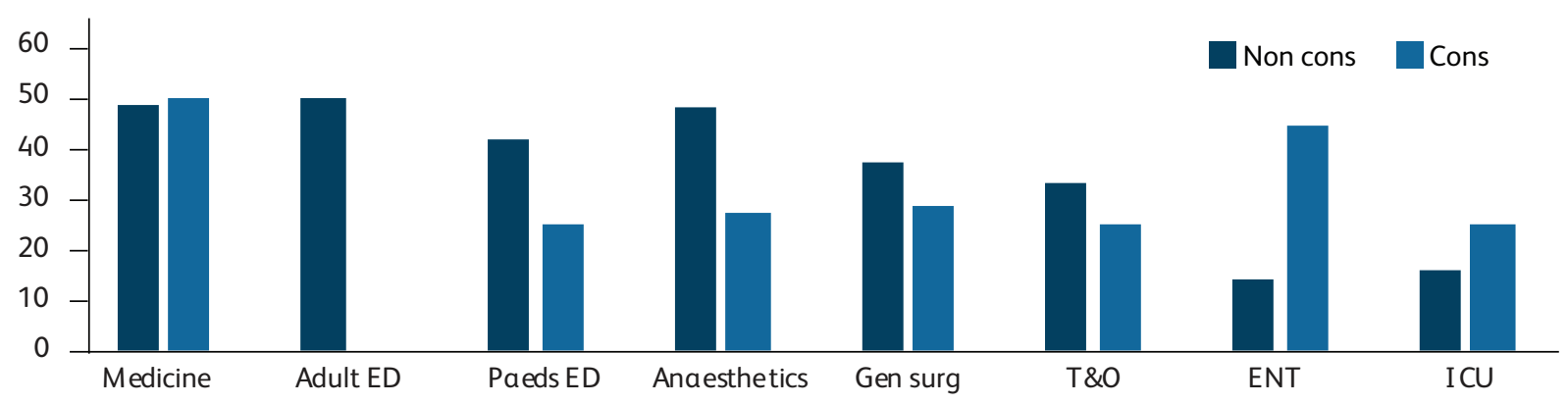

Fig 2. Sickness prevalence by level of seniority. Sickness prevalence (defined as the proportion of doctors that had one or more sickness episode within the period of interest) during period of interest by specialty and grade. Adult ED = adult emergency department; cons = consultants and associate specialists; ENT = ear, nose and throat surgery; ICU = department of intensive care; gen surg = general surgery; medicine = department of medicine and care of the elderly; T\&O = trauma and orthopaedic surgery; non cons = non-consultant grade doctors paeds ED = paediatric emergency department.

Junior doctors had higher sickness prevalence (41.3\%) than seen in senior doctors (32.1\%), although this was not statistically significant $(p=0.137)$. The variation in sickness rates between grades in each department is illustrated in Fig 2.

\section{Discussion}

In a single south London DGH, $39.0 \%$ of doctors working in acute specialities during the COVID-19 pandemic had an episode of sickness over a 6 -week period. The overall sickness absence rate - the proportion of doctors unavailable for work due to sick leave on any given day - was $9.1 \%$. There was variation in incidence of sickness both between different departments, ranging from $17.1 \%$ in ICU to $49.0 \%$ in medicine, and between levels of seniority $32.1 \%$ in senior doctors and $41.3 \%$ in junior doctors.

The doctors in this cohort included almost equal numbers of BAME and white doctors, and of male and female doctors, in keeping with nationwide data. ${ }^{10}$ Sickness absence rates by ethnic background could not be quantified in this study in order to preserve staff confidentiality. The widely reported increased mortality in those of black and Asian ethnicities is particularly concerning for the NHS workforce which has a disproportionately high number of staff from these backgrounds. The reasons for this increased mortality are as yet unknown, although they are likely to include socioeconomic factors, geographical location, prevalence of diabetes and cardiovascular disease, and genetic differences as contributory factors. It is unclear whether BAME people are more likely to catch the disease or are more likely to have severe disease when infected. In addition to sickness from COVID-19, this higher mortality risk is likely to increase anxiety among BAME staff. Various national bodies are presently working on plans to risk assess and mitigate the effects of the virus on BAME staff.

The data included sickness absence for any reason, relating to COVID-19 or not, allowing a full and pragmatic picture of staff sickness levels. Although the reason for absence was not recorded, the markedly higher rate of sickness absence found in this study compared to the NHS England average for January (1.5\%) suggests that COVID-19 has had a large impact, either by causing illness in staff or through national isolation guidance following contact with an unwell household member. It is not possible from these data to draw conclusive reasons for differences in staff sickness rates but this work generates a number of hypotheses related particularly to patient and virus factors, personal protective equipment (PPE), and human factors.

\section{Patient and viral factors}

Differences in the nature of clinical work and patient interaction between specialties may affect sickness rates. The type of patient interaction, the severity of illness, and the time point in a patient's course of illness are all likely to affect risk of transmission.

Different interventions are associated with differing risks of transmission. ${ }^{11}$ Locally, anaesthetists performed many aerosol generating procedures (AGPs), including most endotracheal intubations, and experienced a relatively high level of staff sickness. In contrast, other AGPs were performed in ICU, such as extubation, suctioning, and non-invasive ventilation and sickness rates in ICU doctors were low. While in ED and medicine, much of a doctor's patient interaction involved close contact with someone who may be coughing, in ICU most patients were ventilated on closed circuits with multiple viral filters. High-risk exposure likely varied between grades of doctors in different specialties - traditionally in certain specialties consultants take a more supervisory and managerial role than their juniors, and the extent to which this was maintained during the pandemic may have varied between departments, modifying exposure risk and therefore staff sickness rates.

In a study of serial samples in 31 patients with COVID-19, in severe cases the mean viral load was approximately 60 times higher than that of mild cases and times to viral clearance (negative PCR) were longer. ${ }^{12}$ ED and medicine saw a mixture of mild and severe cases and all of the severe cases were seen in ED and most by medicine before being seen by ICU later in their course, and a proportion not suitable for ICU management remained on the medical wards. In the same study, viral load reduced over the course of a patient's illness. ${ }^{12}$ Patients admitted to ICU are often at a later stage in their disease course which may reduce risk of exposure.

\section{PPE}

Upon their release, Public Health England (PHE) guidelines ${ }^{13}$ on PPE were swiftly adopted, staff were trained on safe use and a good supply of PPE has been available. FFP3 masks, long-sleeved gowns, eye protection and gloves were worn in ICU and the emergency department (ED) resus area as they were identified as high-risk areas where AGPs were regularly performed. In designated ward areas, staff wore surgical masks, aprons, and gloves. Standard PPE, without masks, was worn in areas with low 
COVID-19 risk. Following the collection of this data, surgical masks are now mandatory in all clinical areas.

The guidelines on PPE differ between PHE, the Centers for Disease Control and Prevention (CDC), European Centre for Disease Prevention and Control (ECDC), and the WHO. In contrast with other international bodies, PHE guidelines did not advocate the use of N95/FFP2 masks or long-sleeved gowns in areas with COVID-19 patients. ${ }^{14-16}$ There is weak evidence that surgical masks and N95/FFP2 masks offer similar protection against viral infections in healthcare workers. ${ }^{17,18}$ While pragmatism around PPE stocks and a paucity of evidence for different PPE strategies are likely to have led to divergent guidance, further research and international consensus are needed to reduce transmission risk and staff anxiety. It is reasonable to assume that different PPE guidelines in different clinical areas may have contributed to sickness rate variation between ICU and other hospital doctors, and therefore supports the wider use of FFP3 masks and long sleeve gowns in all areas where there is a risk of COVID-19 transmission.

\section{Human factors}

There is a plethora of social and human factors that contribute to staff sickness rates. A BMA snapshot survey in April found $44 \%$ of doctors said they suffered from anxiety, depression, stress or burnout related to work during the COVID-19 pandemic. ${ }^{19}$ Almost a quarter of staff sickness in the NHS before the pandemic was due to anxiety, stress, depression and other psychiatric illnesses. ${ }^{6}$ Poor staff wellbeing and high burnout rates are also associated with a decline in patient safety. ${ }^{20}$ Higher levels of stress and anxiety could lead to doctors over-reporting sickness.

There may also be positive feedback effects in departments with high levels of sickness. A high sickness rate may stretch the remaining workforce, potentially increasing exposure to the virus and causing increased anxiety. Furthermore, despite attempts to maintain social distancing with the hospital, it is possible that departments with higher sickness rates may have had greater cross-infection between staff.

Greenberg et $a^{21,22}$ have explored the factors that contribute to stress, mental health problems and 'moral injury' among healthcare workers during the COVID-19 pandemic, and have proposed a framework for healthcare leaders in preparing for further COVID-19 surges. They recommend both primary prevention strategies focusing on basic needs (rest areas, PPE etc) and secondary prevention strategies to support traumatised staff (such as the 'trauma risk management' model of peer support and, where necessary, access to frontline mental health professionals with follow-up support).

\section{Planning for the future}

Numbers of COVID-19 patients in UK hospitals are high once again and healthcare leaders must reflect upon the experiences of the first wave. It is imperative to be prepared for further waves but in order to be sustainable as hospitals emerge into a 'new normal', local and national policy must guide the safe resumption of non-emergency services. The excess mortality beyond those dying directly of COVID-19 is a grim reminder of the wide-ranging health impact, much of which is yet to be seen, of focussing the entire health service to a single condition. This raises challenges and should stimulate innovation in how to organise healthcare to protect both patients and staff.
For much of the pandemic, visiting has been extremely limited in hospitals. Resumption of visiting and elective activity will increase staff exposure and careful thought must be given to how best to protect staff. Redesigning patient flow and hospital facilities to allow the greatest degree of social distancing possible is needed. The nature of healthcare often precludes social distancing, and in these circumstances, robust testing and PPE strategies will be crucial. As of 15 June 2020, all staff and visitors in hospitals have been required to wear face coverings. As discussed, PHE guidance on PPE has changed multiple times and as further evidence becomes available and reliable supply lines are re-established, it is possible that these will change again. In particular, our results suggest that full PPE should be available to those working in medicine and ED. As the risk factors associated with severe COVID-19 become increasingly clear, hospital management has a duty to use this time to ensure staff are risk assessed and consider redeployment to lower risk areas as appropriate.

Testing policy and infrastructure for staff has evolved significantly over this time period and, going forward, may have important implications for staff sickness rates in allowing people to return to work sooner if their COVID-19 polymerase chain reaction (PCR) swab is negative. Rapid turnaround or even point of care testing could allow risk stratification of patients and facilitate serial testing of staff to reduce cross-infection and avoidable sickness absence. Hospitals should consider redeployment of staff to cover sickness, with a higher number of doctors covering departments hardest hit by sickness rates, such as medicine. While many hospitals undertook widespread redeployments to specialties directly involved in the care of COVID-19 patients, the need for ongoing provision of other services is increasingly apparent and, as seen in this study, surgical specialties also experienced significant rates of sickness absence. During the 'first wave' there was an understandable focus on coping with COVID-19 in ED, medicine, and ICU. If the pandemic persists for a significant period of time it will be imperative to balance safe staffing requirements across services and hospitals should review their absence data to help them to plan required levels of redundancy. One might hope that staff sickness levels would be reduced in a second wave if staff have developed immunity although the extent and duration of this is not currently understood.

\section{Limitations}

Although basic departmental demographic data were collected, sickness episodes were not associated with demographic data to preserve confidentiality. Furthermore, a range of relevant variables such as age, pre-existing health conditions, mode of commute, and number of household members were not collected. It is likely that differences in these factors between specialties or grades may have confounded our observations.

The reason for each sickness event was not recorded. As such it is not clear whether staff were unwell with symptoms consistent with COVID-19, another illness, or were isolating following contact with an unwell household member. While this would be of interest, the implications for those responsible for workforce logistics are less important. This study sought to pragmatically and anonymously identify the overall impact of the COVID-19 pandemic on interdepartmental staffing.

Full sickness episodes were included if they overlapped either end of the study period. This did not affect the percentage of staff 
with a sickness episode but may have led to a small overestimate in the sickness absence rate.

Since the primary focus of the study was sickness absence among doctors, extrapolation of these findings to the many clinical and non-clinical staff who contribute to patient care cannot be assumed. Sickness absence rates in doctors likely underestimate the rate in the wider NHS workforce as in prepandemic times, doctors have the lowest sickness absence rate of any healthcare workers. ${ }^{6}$ Further study in these key staff groups as well as in allied health professionals and other hospital staff, including non-patient facing roles, would provide a more complete picture.

\section{Conclusion}

Staff sickness rates among frontline doctors during the first wave of COVID-19 were high in a south London DGH. There was marked variation between departments and some between different grades of doctor. Those responsible for rapidly expanding and redeploying the hospital workforce during the COVID-19 pandemic can use these observations to inform healthcare delivery and to guide their preparation for further waves.

\section{Supplementary material}

Additional supplementary material may be found in the online version of this article at www.rcpjournals.org/clinmedicine: S1 - Baseline characteristics of departments by number (percentage) of doctors

\section{References}

1 World Health Organization. WHO Timeline - COVID-19. WHO, 2020. www.who.int/news-room/detail/27-04-2020-who-timeline--covid-19 [Accessed 1 May 2020].

2 European Centre for Disease Prevention and Control. Coronavirus disease 2019 (COVID-19) in the EU/EEA and the UK - eighth update. ECDC, 2020. www.ecdc.europa.eu/sites/default/files/ documents/covid-19-rapid-risk-assessment-coronavirus-disease2019-eighth-update-8-april-2020.pdf.

3 Office for National Statistics. Coronavirus (COVID-19 related deaths by occupation, England and Wales: deaths registered between 9 March and 28 December 2020. www.ons.gov.uk/peoplepopulationandcommunity/health ndsocialcare/causesofdeath/bulletins/.

4 Scavone F. Doctors still without adequate supplies of PPE, major BMA survey finds. BMA, 2020. www.bma.org.uk/news-and-opinion/ doctors-still-without-adequate-supplies-of-ppe-major-bma-surveyfinds-1 [Accessed 22 May 2020].

5 NHS Digital. NHS vacancy statistics England February 2015 December 2019, experimental statistics. NHS Digital, 2020. https:// digital.nhs.uk/data-and-information/publications/statistical/ nhs-vacancies-survey/february-2015---december-2019-experimentalstatistics.

6 NHS Digital. NHS sickness absence rates - January 2020, Provisional Statistics. NHS Digital, 2020. https://digital.nhs.uk/dataand-information/publications/statistical/nhs-sickness-absence-rates/ january-2020-provisional-statistics [Accessed 22 May 2020].

7 Hancock M. Health and social care secretary's statement on coronavirus (COVID-19): 2 April 2020. GOV.UK. DHSC, 2020. www. gov.uk/government/speeches/health-and-social-care-secretarysstatement-on-coronavirus-covid-19-2-april-2020 [Accessed 1 May 2020].
8 Royal College of Physicians. COVID-19 and its impact on NHS workforce. RCP, 2020. Available from www.rcplondon.ac.uk/news/ covid-19-and-its-impact-nhs-workforce [Accessed 1 May 2020].

9 Intensive Care National Audit and Research Centre. ICNARC report on COVID-19 in critical care 08 May 2020. ICNARC, 2020. Available from: www.icnarc.org/Our-Audit/Audits/Cmp/Reports.

10 NHS Digital. NHS workforce statistics - March 2019 (Including supplementary analysis on pay by ethnicity). NHS Digital, 2020 Available from: https://digital.nhs.uk/data-and-information/ publications/statistical/nhs-workforce-statistics/nhs-workforcestatistics---march-2019-provisional-statistics [Accessed 17 ]une 2020].

11 Harding H, Broom A, Broom J. Aerosol generating procedures and infective risk to healthcare workers: SARS-CoV-2 - the limits of the evidence. J Hosp Infect 2020;105:717-25.

12 Liu Y, Yan L-M, Wan L et al. Viral dynamics in mild and severe cases of COVID-19. Lancet Infect Dis 2020;20:656-7.

13 Public Health England. COVID-19 personal protective equipment (PPE). PHE, 2020. www.gov.uk/government/publications/ wuhan-novel-coronavirus-infection-prevention-and-control/ covid-19-personal-protective-equipment-ppe [Accessed 1 May 2020].

14 World Health Organization. Infection prevention and control during health care when novel coronavirus ( $\mathrm{nCoV}$ ) infection is suspected. WHO, 2020. Available from: www.who.int/publications-detail/ infection-prevention-and-control-during-health-care-when-novelcoronavirus-(ncov)-infection-is-suspected-20200125 [Accessed 1 May 2020].

15 Centers for Disease Control and Prevention. Coronavirus Disease 2019 (COVID-19) 2020. CDC, 2020. www.cdc.gov/coronavirus/ 2019-ncov/hcp/infection-control-recommendations.html [Accessed 1 May 2020].

16 European Centre for Disease Prevention and Control. Guidance for wearing and removing personal protective equipment in healthcare settings for the care of patients with suspected or confirmed COVID-19. ECDC, 2020. www.ecdc.europa.eu/sites/default/files/ documents/COVID-19-guidance-wearing-and-removing-personalprotective-equipment-healthcare-settings-updated.pdf.

17 Bartoszko J], Farooqi MAM, Alhazzani W, Loeb M. Medical masks vs N95 respirators for preventing COVID-19 in healthcare workers: A systematic review and meta-analysis of randomized trials. Influenza Other Respir Viruses 2020;14:365-73.

18 Long Y, Hu T, Liu L et al. Effectiveness of N95 respirators versus surgical masks against influenza: A systematic review and metaanalysis. J Evid Based Med 2020;13:93-101.

19 McArdle J. Stress and burnout warning over COVID-19. BMA, 2020. www.bma.org.uk/news-and-opinion/stress-and-burnout-warningover-covid-19 [Accessed 22 May 2020].

20 Hall LH, Johnson J, Watt I et al. Healthcare staff wellbeing, burnout, and patient safety: a systematic review. PLoS One 2016;11: e0159015.

21 Greenberg N, Tracy D. What healthcare leaders need to do to protect the psychological well-being of frontline staff in the COVID-19 pandemic. Leader 2020;4:101-2.

22 Greenberg N, Docherty M, Gnanapragasam S, Wessely S. Managing mental health challenges faced by healthcare workers during covid-19 pandemic. BMJ 2020;m1211.

Address for correspondence: Dr Reza Khorasanee, University Hospital Lewisham, High Street, Lewisham, London SE13 6LH, UK.

Email: reza.khorasanee@nhs.net 